\title{
Degeneration of muscle in association with carcinoma of the bronchus
}

\author{
BETTY BROWNELL AND J. T. HUGHES \\ From the Burden Neuropathological Laboratory, Frenchay Hospital, Bristol, \\ and the Department of Neuropathology, Radcliffe Infirmary, Oxford
}

SYNOPSIS The clinical features and necropsy findings are described in three cases in which severe and rapidly progressive muscle weakness developed in association with carcinoma of the bronchus. In all three cases, muscular weakness was directly responsible for death. Histological and ultramicroscopical examination in all cases showed an unusual type of degeneration of muscle fibres accompanied by degeneration of intramuscular nerve fibres, but without involvement of the central nervous system, or of the peripheral nerve trunks. The findings are compared with those of previously reported cases, and possible mechanisms for the muscle degeneration are discussed.

Many neurological and neuromuscular syndromes have now been ascribed to nonmetastatic effects of carcinoma (Brain and Adams, 1965; Henson, 1969) and these clinical syndromes are associated with pathological changes involving brain, spinal cord, peripheral nerve, or muscle. Probably the muscle disorder most commonly associated with carcinoma is the myasthenic-myopathic syndrome, in which the pathological changes in muscle are nonspecific and mild in relation to the clinical disability (Henson, 1969). Polymyositis may also occur in patients with carcinoma, and in these cases the clinical and pathological features are indistinguishable from those of idiopathic polymyositis (Adams et al., 1962; Adams, 1969). Denervation atrophy of muscle has also been reported in carcinomatous syndromes, caused either by peripheral neuropathy or by the socalled carcinomatous motor neurone disease (Brain et al., 1965; Shy and Silverstein, 1965).

Acute necrotizing myopathy associated with carcinoma was first described by Barbara Smith $(1968,1969)$ in reports of three cases, and subsequently by Urich and Wilkinson (1970). This paper presents the clinical features and necropsy findings in three cases similar to those described by these authors.

(Accepted 31 October 1974.)

\section{METHODS}

Paraffin sections stained by routine methods were prepared from postmortem tissues, and for the examination of intramuscular nerve fibres, the method of Schofield (1960) was applied to frozen sections. For electron microscopy, tissue which had been stored in $10 \%$ formalin for two months was used; small pieces of the muscle were washed in buffer, post-fixed in $1 \%$ osmium tetroxide, and washed again in buffer, before being dehydrated in successive alcohol solutions and embedded in Araldite.

\section{CASE 1}

Female, aged 72 years (NP.1970). This lady presented with a history of a cough for four weeks and chest radiograph showed a tumour of the right upper lobe of the lung. She was given a course of radiotherapy, after which the mass had apparently resolved and she was well. One week later she was admitted to hospital because she had rapidly developed weakness of her arms and difficulty in walking.

On examination, the cranial nerves were intact but motor power was symmetrically reduced in all muscle groups in the upper and lower limbs. Sensation was normal, tendon reflexes equal and normal, and plantar responses were flexor. The results of electromyography (which was a very limited examination on account of the patient's severe weakness and lack of cooperation) were suggestive of myopathy. 


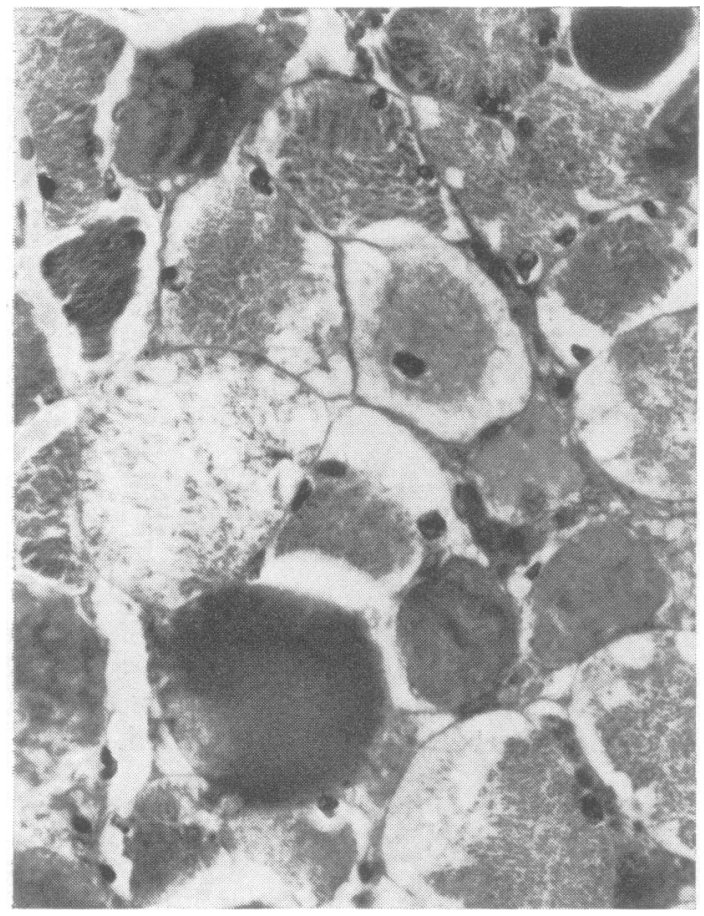

FIG. 1 Case 1. Photomicrograph of section of deltoid muscle, showing degeneration of muscle fibres. Haematoxylin and eosin, $\times 400$.

The serum creatine phosphokinase activity was $\mathbf{1 2 . 9}$ $\mu \mathrm{mol}$ per ml per hour (normal being less than 1.5 $\mu \mathrm{mol}$ per ml per hour). Barium swallow revealed abnormalities compatible with neuromuscular incompetence.

Her illness progressed rapidly and there was no response to cortisone therapy. She died of aspiration pneumonia four weeks after her admission to hospital, and five weeks after the onset of her muscular weakness.

NECROPSY FINDINGS The right upper lobe bronchus was occluded by a small tumour mass, and there was extensive bronchopneumonia in both lungs. Metastatic tumour deposits were present in the liver. The thyroid gland was small, nodular, and fibrous. The muscles of the upper and lower limbs and of the trunk were abnormally pale and soft. Histological examination of the tumour in the lung showed a dense fibrous scar, due to radiation effect, with only a few clumps of large hyperchromatic tumour cells. Sections of the liver secondaries showed an anaplastic carcinoma of oat-cell type. The histological

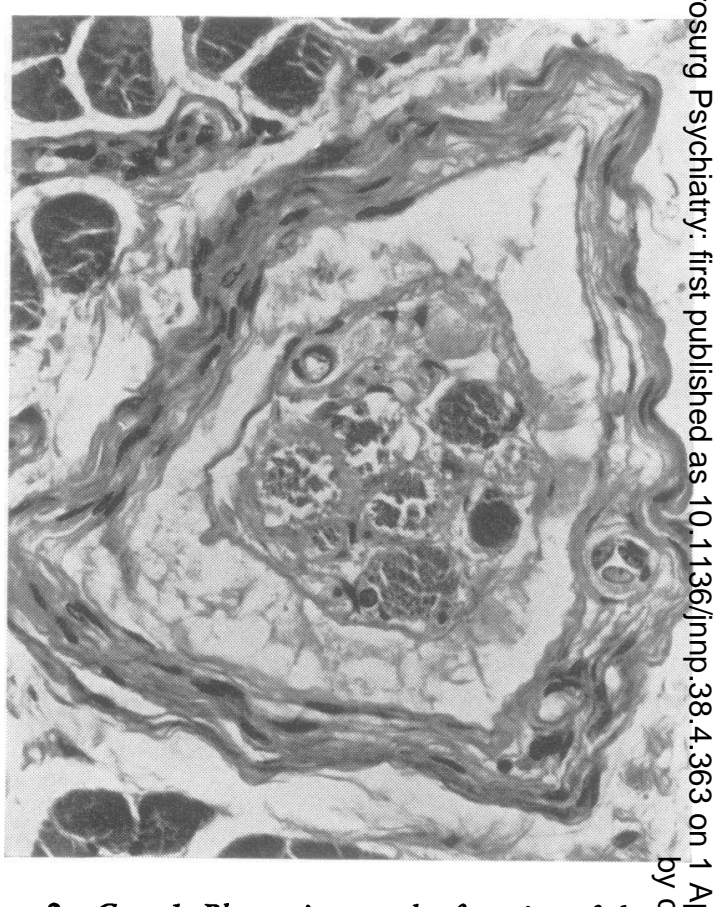

FIG. 2 Case 1. Photomicrograph of section of thenair muscle, showing degeneration of intrafusal fibres in muscle spindle. Haematoxylin and eosin, $\times 260$.

appearances of the thyroid were those of a burnt out Hashimoto's disease.

NERVOUS SYSTEM The brain and spinal cord were $\overrightarrow{\overrightarrow{0}}$ examined after fixation in formalin. Neither showed 3 any naked-eye abnormalities, and sections of the spinal cord at various segmental levels showed no: evidence of anterior horn cell disease or of long tract degeneration. The spinal nerve roots and lumbar: posterior root ganglia were normal.

MUSCles The tongue, deltoid, biceps brachii, thenar, sartorius, and rectus femoris muscles were examined. All the muscles except the tongue showed윽 disease, equally severe in proximal and distal $>$ muscles and in upper and lower limbs. The most prominent feature was extensive degeneration of N muscle fibres, of granular or vacuolar type, within the intact sarcolemma (Fig. 1). The distribution of ${ }^{N}$ the degenerating fibres was random, without motor unit pattern, and, in the worst affected areas, more than $50 \%$ of muscle fibres were affected. Inflamma-e tory cell reaction to the widespread degeneration $\mathbb{D}$ 


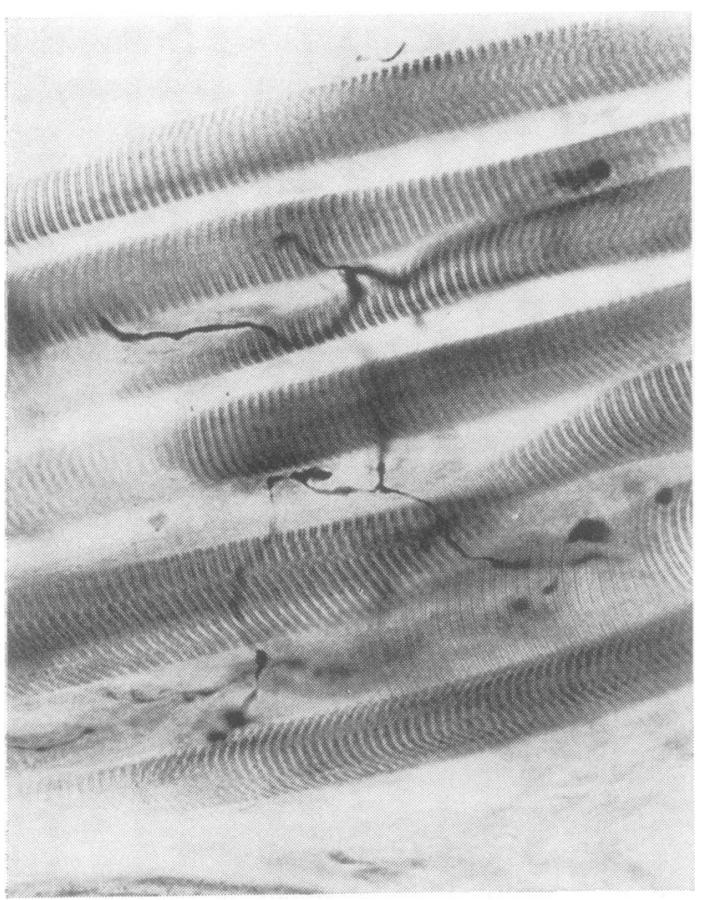

FIG. 3 Case 1. Photomicrograph of section of deltoid muscle, showing branching of terminal nerve fibres. Schofield's modification of Bielschowsky's stain, $\times 400$.

was almost entirely absent, and phagocytosis of necrotic muscle was only rarely present. Muscle spindles appeared to be similarly affected by the disease process, showing degeneration of intrafusal fibres within their intact capsules (Fig. 2). Stains for nerve axons revealed a widespread, severe, active degeneration of terminal nerve fibres with droplet degeneration of axons, and axonal swellings. In addition, striking regenerative changes were present, in the form of fine, branched, sometimes beaded fibres with multiple end-plates on single muscle fibres (Fig. 3).

ELECTRON MICROSCOPY Grids from four separate portions of muscle were examined. The necrotic muscle fibres were recognized in $1 \mu \mathrm{m}$ thick sections using the light microscope and then with the electron microscope at low magnifications. It was clear that these abnormal muscle fibres were affected by a severe form of necrosis, and higher magnifications showed that most of the formed elements had dis-

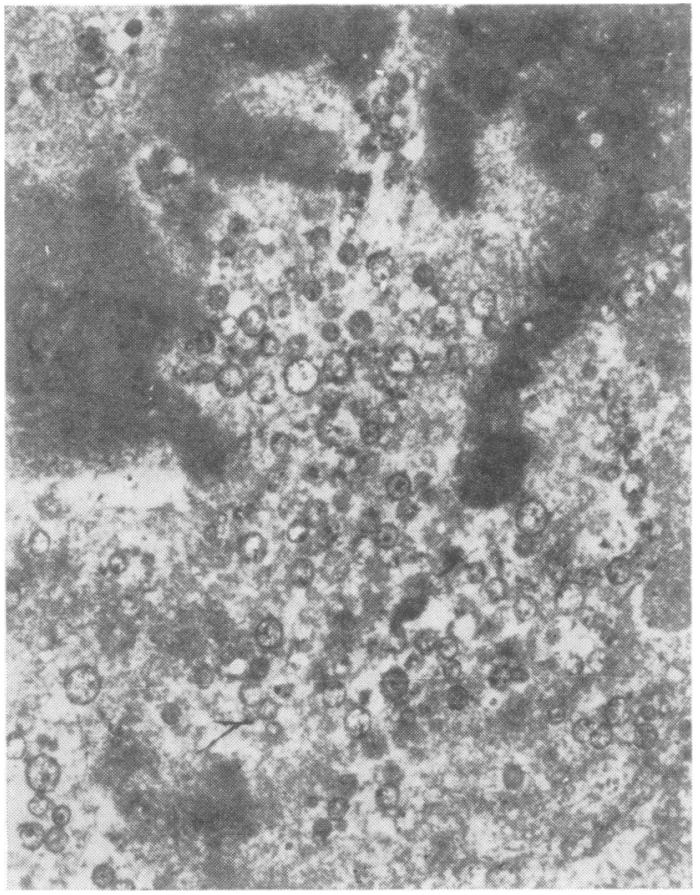

FIG. 4 Case 1. Electron micrograph at low magnification of formalin-fixed deltoid muscle, showing a peripheral area of a degenerating muscle fibre. Most of the formed elements have disappeared and the only recognizable organelles are mitochondria. Two per cent aqueous uranyl acetate/lead citrate, $\times 6000$.

appeared from the necrotic muscle fibres (Fig. 4). No myofilaments could be recognized and no band structure was apparent. No tubules or sacs of endoplasmic reticulum were present. The inner membrane of the sarcolemma had degenerated leaving only the outer basement membrane as the boundary of the cell. The muscle cell contained a finely fibrillar electron-dense material suggestive of denatured protein and the only formed elements seen were mitochondria, which were numerous and arranged irregularly throughout the cell. No sarcolemmal nuclei were seen in the necrotic muscle cells. No inflammatory cells were found either in or around the muscle fibres.

PERIPHERAL NERVES The femoral, median and radial nerves were not significantly abnormal.

\section{CASE 2}

Female, aged 63 years (NP.2363). This patient was 


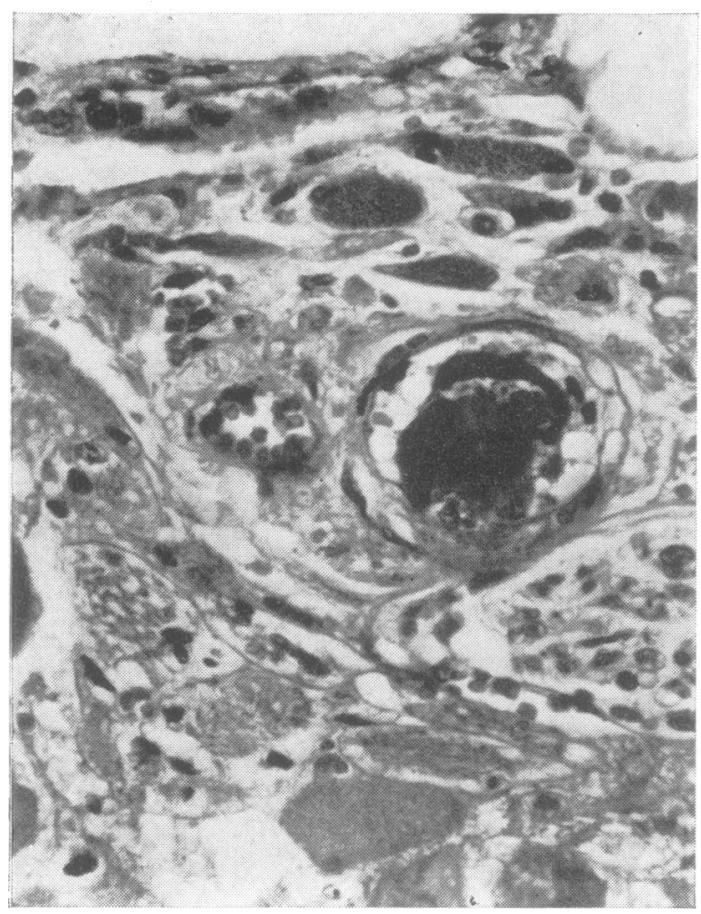

FIG. 5 Case 2. Photomicrograph of section of biceps brachii muscle, showing degeneration of muscle fibres. Regenerating fibres are also present. Haematoxylin and eosin, $\times 400$.

admitted to hospital as a medical emergency, because of rapidly progressive weakness of her arms and legs, accompanied by aching pain. She had no sensory symptoms, nor were there any symptoms referable to any other systems.

On examination, her general condition was good but she was noted to have a plethoric face and to have a blood pressure of $150 / 80 \mathrm{mmHg}$. Neurological examination revealed no abnormality of the cranial nerves, but there was considerably diminished power in all muscle groups of both upper and lower limbs and the trunk which was symmetrical and involved proximal and distal muscles alike. Sensation was entirely normal, tendon reflexes were present and equal, and plantar reflexes were flexor. Radiology of the chest carried out on the day after admission revealed a moderately large opacity in the left lung, and she was therefore transferred to the thoracic surgery unit for operation. By this time she had become extremely weak and had developed dysphagia. Three days after her admission, a left pneumonectomy was performed with the finding of

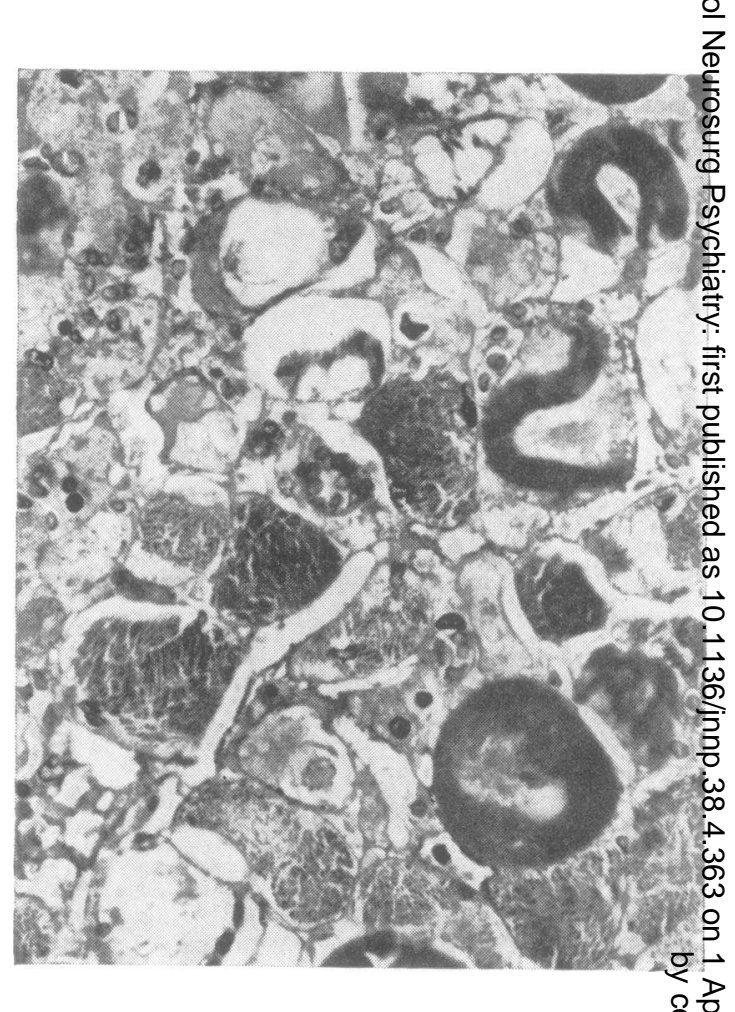

FIG. 6 Case 3. Photomicrograph of section of delt muscle, showing degeneration of muscle fibr: $\overrightarrow{0}$ Haematoxylin and eosin, $\times 400$.

a tumour in the dorsal segment of the lower lobe. $\frac{\square}{\varnothing}$ Histology of the tumour revealed an anaplastic oat- $\supseteq$ celled carcinoma with involvement of the peri- $\overrightarrow{\overrightarrow{0}}$ bronchial lymph nodes. The patient tolerated the operation reasonably well but her muscle weakness was unaltered and during the following week she became more and more weak and eventually required artificial respiration. She died 10 days after? the operation, 25 days after her first admission to $\frac{\text { 을 }}{3}$ hospital.

NECROPSY FINDINGS There was severe, extensive bronchopneumonia in the right lung. The cardio-을 vascular, gastrointestinal, genitourinary, and reticu- $D$ loendothelial systems were all normal. No residual을. or metastatic tumours were found.

CENTRAL NERVOUS SYSTEM The brain showed no $\mathrm{O}$ naked eye lesions. The spinal cord was not examined. స్

MUSCles The tongue, diaphragm, deltoid, bicepsco brachii, thenar, lumbrical, vastus lateralis, rectus $\frac{\bar{\Phi}}{\mathscr{C}}$ 


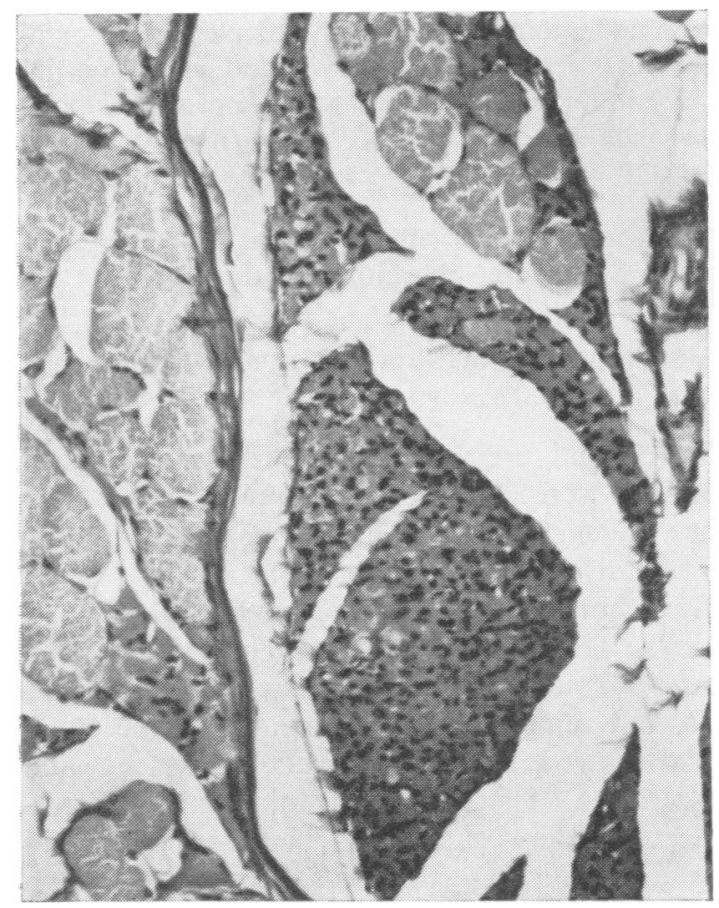

FIG. 7 Case 3. Photomicrograph of section of peroneus longus muscle, showing neurogenic atrophy. Haematoxylin and eosin, $\times 180$.

femoris, and peroneus longus muscles were examined. All muscles except the tongue showed an extensive, though patchy, necrotizing myopathy. Affected muscle fibres were either swollen or shrunken, with vacuolar, hyaline, or granular degeneration, often present within an intact sarcolemmal membrane (Fig. 5). In the most severely affected areas, sarcolemmal nuclei had disappeared, but here and there nuclei had proliferated, occasionally forming chains, and a few basophilic regenerating muscle fibres were also seen. A few inflammatory cells were present, but did not appear to be a prominent feature of the lesions. Large monocytes were not present, and there was no phagocytosis of necrotic muscle fibres. The disease process appeared to include the intrafusal fibres of many of the muscle spindles. The intramuscular arteries looked normal and no tumour cells were seen. Sections stained for nerve axons showed a severe and widespread acute distal neuropathy, with extensive degeneration, and some regenerative changes.

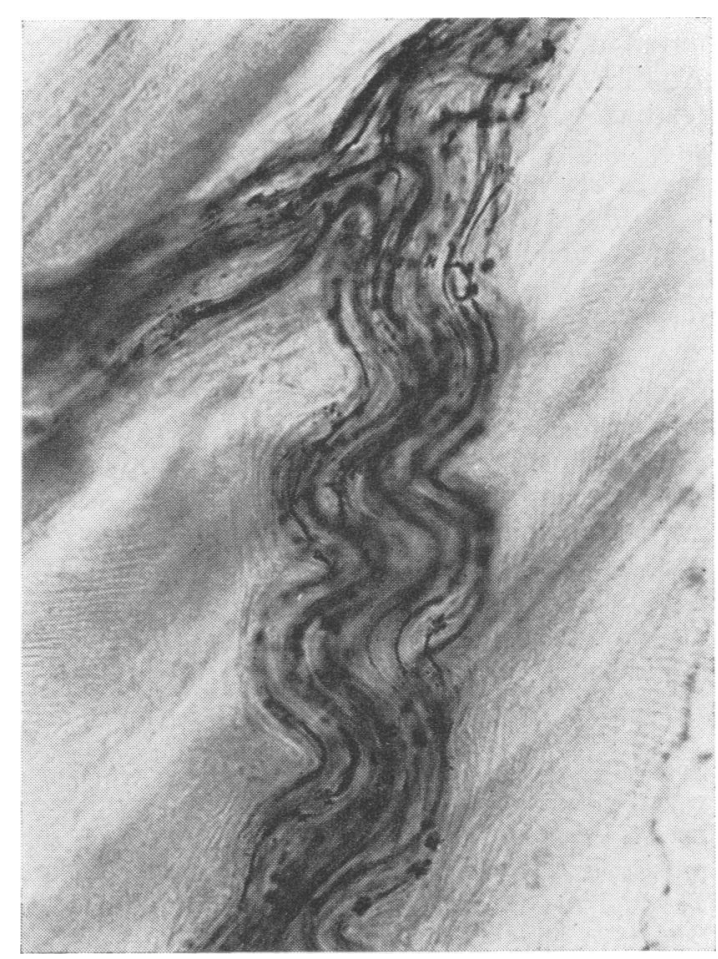

FIG. 8 Case 3. Photomicrograph of section of deltoid muscle, showing degeneration of intramuscular nerve axons. Schofield's modification of Bielschowsky's stain, $\times 400$.

ELECTRON MICROSCOPY Many muscle fibres were undergoing necrosis and these stood out in contrast to the normal fibres. In the degenerating fibres the myofilaments were depleted but not completely absent as in case 1 . The band structure was in disarray and portions of displaced Z-band protein could be recognized. Mitochondria were numerous throughout the muscle cell. The sarcolemmal nuclei were absent and the plasma membrane of the sarcolemma had degenerated.

PERIPHERAL NERVES The ulnar, median, femoral, and sciatic nerves were not significantly abnormal.

\section{CASE 3}

Male, aged 56 years (NP.2380). This man presented with six weeks' history of weakness, first of the lower and, later, of the upper limbs. After numerous investigations, a diagnosis was made of pituitary dependent Cushing's disease and he was then re- 
ferred to the neurosurgical unit for hypophysectomy. At this stage, he was found to be plethoric with features of Cushing's syndrome, with a blood pressure of $180 / 100 \mathrm{mmHg}$. Examination of the cranial nerves revealed no abnormality. Severe weakness was present in proximal and distal muscles of the upper and lower limbs, and in the trunk. Tendon reflexes were normal, plantar reflexes flexor, and there were no sensory changes.

A transethmoidal hypophysectomy was carried out the day after admission, histology of the excised pituitary showing normal anterior and posterior pituitary gland tissue, with no evidence of tumour. After operation, the patient's muscle weakness was no better, and over the following three weeks he became extremely weak, developed respiratory paralysis, and ultimately bronchopneumonia. He died four weeks after the hypophysectomy, three months after the onset of his muscle weakness.

NECROPSY FINDINGS A small, primary carcinoma was present near the origin of the right middle lobe bronchus, and metastatic tumour deposits were present in the liver and in the right suprarenal gland. Throughout the body there was a generalized increase in the amount of adipose tissue, and the pancreas showed fat necrosis. Muscles of upper and lower limbs were abnormally pale. Histological examination showed the lung tumour to be an anaplastic, oat-celled carcinoma.

CENTRAL NERVOUS SYSTEM The brain and spinal cord were examined after fixation in formalin. The brain showed no significant abnormalities. Sections of the spinal cord revealed no evidence of anterior horn cell disease or of long tract degeneration, and no abnormalities were present in nerve roots or posterior root ganglia.

MUSCLES The tongue, deltoid, flexor carpi ulnaris, thenar, quadriceps, sartorius, and peroneus longus muscles were examined, all but the tongue showing extensive degeneration of muscle fibres (Fig. 6), the sarcoplasm apparently degenerating within an intact sarcolemma. Muscle spindle fibres were also affected. No inflammatory cells were present, and phagocytosis of the necrotic fibres was not seen. Although the degenerating fibres had a random distribution, there was, in addition, motor unit atrophy, from which it was concluded that denervation atrophy had occurred (Fig. 7). Nerve fibre stains on all affected muscles revealed a very severe and widespread acute distal neuropathy, without any evidence of regeneration (Fig. 8).
ELECTRON MICROSCOPY Some of the muscle fibres were undergoing necrosis with depletion of myofilaments, irregularity of the band structure, in-e crease in mitochondria, and absence of sarcolemmal o nuclei and the plasma membrane of the sarcolemma. The appearances were very similar to those in case 2 .

PERIPHERAL NERVES The femoral, median, and ulnar nerves were substantially normal.

\section{SUMMARY OF CLINICAL AND PATHOLOGICAL FEATURES}

The clinical presentation and the pathological findings were similar in all three cases. In each $\rightarrow$ case the clinical presentation was with a rapidly progressive muscle weakness which caused death $\vec{\omega}$ after short illnesses lasting five, six, and 12 ? weeks. The weakness appeared to involve all? muscle groups, being first apparent as limb weak- $\dot{\omega}_{\infty}$ ness but rapidly spreading to involve the trunk muscles and the muscles of swallowing and $\dot{\sigma}^{\prime}$ respiration. There were no physical signs im- $\omega$ plicating the central or peripheral nervous systems. Despite the weakness the tendo on $\rightarrow$ reflexes were always preserved. All three cases had a carcinoma of the bronchus. Case 2 and case 3 had features of Cushing's disease and 宙 case 3 there were endocrine manifestations of $\cdot$ secretory carcinoma of the bronchus.

The pathological findings in each case were also striking and consistent. All the muscles examined were abnormal except for the tongue. The common finding in all three cases was degeneration of muscle fibres without phago- $\frac{\overline{0}}{3}$ cytosis and with a minimal inflammatory reaction. The intrafusal fibres within the muscle spindles were similarly affected by degeneration. All cases showed acute degeneration of terminalin-흠 tramuscular nerve fibres, and denervation atrophy $\dot{0}$ of the muscle was detectable in case 3 . Regeneration phenomena could be seen in muscle fibres $ᄋ$ (case 2), and in terminal nerve fibres (cases 1 and 2). No tumour cells were found in any of the $\frac{}{5}$ muscles, and no significant disease or occlusion $\frac{D}{O}$ of intramuscular arteries could be detected. Electron microscopical examination showed pro- $N$ found necrosis of the affected muscle fibres, $N$ which lacked all the contractile protein and had N no band structure or tubular systems. Mitochondria were the only organelles preserved.

No significant pathological changes were found 
in the central nervous system, and, in particular, in the two cases where the spinal cord was examined (cases 1 and 3) anterior horn cells, spinal nerve roots, and posterior root ganglia were normal. Examination of peripheral nerve trunks revealed no significant abnormalities.

In all three cases, the primary tumour was an anaplastic bronchial carcinoma of oat-cell type, without metastases in case 1 , and with insignificant metastases (in relation to the muscle disorder) in cases 2 and 3.

\section{DISCUSSION}

The three cases reported here represent an uncommon type of muscle necrosis occurring in association with carcinoma of the bronchus. These cases are comparable with four previously reported cases which will now be briefly cited.

Smith $(1968,1969)$ described the clinical features and pathological findings in muscle biopsies from three cases. Case 1 was a 54 year old woman who had been treated for carcinoma of the colon by a colectomy operation, after which she had rapidly developed a syndrome of muscle weakness. Case 2 was a 60 year old woman with a carcinoma of the left breast who developed weakness of her hands and arms associated with skin manifestations. After a mastectomy, the muscular weakness and cutaneous eruptions regressed, and two years later the patient was alive and well with no weakness. Case 3 was a 48 year old woman with a carcinoma of the stomach who developed mild proximal muscle weakness and a rash. She died from her tumour which had proved inoperable at laparotomy. The muscle biopsies on all these three cases showed extensive necrosis of the muscle fibres, apparently taking place within the sarcolemma, and leaving this structure intact. There was very little inflammatory response, and phagocytosis by histiocytes was not prominent. Regeneration of muscle fibres was present in all the cases. Muscle innervation was described only in case 1 , in which methylene blue staining of the terminal innervation showed normal findings.

The histological features in our own three cases are very similar to those described by Smith. However, we found severe degeneration of the terminal nerve fibres which was not seen by Smith in the one case in which nerve fibres were examined. In their clinical aspects, our cases differ in that the muscle disorder was more fulminating, and probably more significant in causing death than in Smith's cases. In two of our cases, treatment of the primary carcinoma, by irradiation or excision, had no beneficial effect on the muscle disease. In Smith's case 2 the myopathy improved when the carcinoma of the breast had been removed but in none of the other cases was any improvement in the muscle disease seen when the malignant growth was treated. Case 1 of Smith was exceptional in that the myopathy developed after the tumour had been removed.

Urich and Wilkinson (1970) described a case of muscle necrosis in a 59 year old man with a carcinoma of the stomach; this patient had two muscle biopsies and ultimately a full necropsy. The clinical features were very similar to those of our cases; with rapidly advancing muscle weakness including impairment of swallowing and respiration. The histological changes described in the muscles (at necropsy) of this case were almost identical with those of our cases but the state of the intramuscular nerves was not reported.

Our cases and those reviewed above provide a body of evidence linking carcinoma-in our three cases carcinoma of the bronchus-with a syndrome of muscle degeneration. Our pathological descriptions have emphasized the microscopical and ultramicroscopical changes in the muscle which are, in our experience, very unusual.

No explanation for the association of myopathy with carcinoma has yet been proven, though several can be suggested. The invasion of muscle by a virus, taking advantage of an altered immunological state, is a possibility, but one for which no positive evidence is available. Virus-like particles have been reported in cases of polymyositis (Hughes and Esiri, in preparation) but in none of these reported cases was there an associated carcinoma. We have, in the three cases reported here, conducted an extensive search by electron microscopy on portions of many of the affected muscles. No structures resembling virus particles were seen in any of the tissues examined.

The existence of an altered immune state in which self destruction of muscle is caused by an autoimmune mechanism requires consideration. 
We have a model of this situation in human polymyositis (some cases of which are associated with carcinoma) and also in experimentally produced autoimmune polymyositis (Dawkins, 1965). In these two conditions the histological appearances differ markedly from those seen in the cases described here. Notably, the microscopical appearances in our cases lack the extensive lymphocytic infiltration which, from experimental and other evidence, we now know to be invariably present and probably causative in autoimmune polymyositis (Esiri et al., 1973). Whether a humoral autoimmune system could cause this disease is doubtful, but in the known autoimmune polymyositis this type of immune response does not appear to be important.

It is conceivable that a carcinoma of the bronchus could produce an active circulating substance which is myotoxic. Such specific toxins are known in biology-for example, the venom of the Beaded Seasnake, Enhydrina schistosa (Reid, 1961) - but as yet we have no evidence for any such toxin produced by a human cell.

A more acceptable explanation involves the production of a hormone by the tumour, and of course hormone secretion is well recognized in some cases of carcinoma of the bronchus. It is interesting and probably highly significant that in two of our three cases there was evidence that the tumours were hormonally active. The pattern of hormonal activity produced the clinical features of Cushing's disease in both these cases and in case 3 there were other hormonal features of secretory carcinoma of the bronchus. How these various hormones might cause muscle necrosis is difficult to understand, but it is possible that an effect on the vessels of muscles could cause ischaemia and muscle infarction. The histological appearances would agree very well with this explanation for which there is, however, no direct evidence. No single active hormone can be named which might have this effect.

A paper by Heffner (1971) describes three cases of muscle necrosis associated with adenocarcinoma of the gastrointestinal tract. Necropsies on these cases revealed that the cause of the muscle necrosis was arterial occlusion by emboli, and in all cases there was endocarditis (nonbacterial) of one of the heart valves, as well as evidence in other organs of embolic phenomena. In the light of this report we have re-examined $\stackrel{\oplus}{\subseteq}$ all the histological slides of our three cases. No vascular occlusion of any type could be found in any of the numerous muscles examined, and we conclude that Heffner's cases have a different aetiology from that of the three cases described. in this paper.

We are indebted to physicians and surgeons of Frenchay Hospital, Bristol, for our clinical material, and we thank o Mrs K. Schomberg and Mr A. W. Churchill for technical and photographic assistance.

\section{REFERENCES}

Adams, R. D. (1969). Pathological reactions of the skeletal $\vec{\omega}$ muscle fibre in man. In Disorders of Voluntary Muscle, pp. 143-202. 2nd edn. Edited by J. N. Walton. Churchill: London.

Adams, R. D., Denny-Brown, D., and Pearson, C. M. (1962). ic Diseases of Muscle, pp. 435-436, 2nd edn. Harper: New $@$ York.

Brain, Lord, and Adams, R. D. (1965). Epilogue: A guide to the classification and investigation of neurological dis- $\omega$ orders associated with neoplasms. In The Remote Effects of $\mathrm{O}$ Cancer in the Nervous System, pp. 216-221. Edited by Lord Brain and F. H. Norris. Grune and Stratton: New York.

Brain, Lord, Croft, P. B., and Wilkinson, M. (1965). Moter neurone disease as a manifestation of neoplasm. Brain, $\overrightarrow{0 .} \overrightarrow{0}$

Dawkins, R. L. (1965). Experimental myositis associate or with hypersensitivity to muscle. Journal of Pathology and Bacteriology, 90, 619-625.

Esiri, M. M., MacLennan, I. C. M., and Hazleman, B. L. (1973). Lymphocyte sensitivity to skeletal muscle in $\bar{\partial}$ patients with polymyositis and other disorders. Clinical ڤ and Experimental Immunology, 14, 25-35.

Heffner, R. R., Jr (1971). Myopathy of embolic origin in 2 patients with carcinoma. Neurology (Minneap.), 21, 840- $\overrightarrow{\overline{0}}$ 846.

Henson, R. A. (1969). Neuromuscular disorders associated with malignant disease. In Disorders of Voluntary Muscle, pp. 639-652, 2nd edn. Edited by J. N. Walton. Churchill: London.

Reid, H. A. (1961). Myoglobinuria and sea-snake-bite poisoning. British Medical Journal, 1, 1284-1289.

Schofield, G. C. (1960). Experimental studies on the innervation of the mucous membrane of the gut. Brain, 83, 490514.

Shy, G. M., and Silverstein, I. (1965). A study of the effects upon the motor unit by remote malignancy. Brain, 88, 515-528.

Smith, B. (1968). Skeletal muscle necrosis associated with carcinoma. In Research in Muscular Dystrophy: Proceed- $\frac{1}{2}$ ings of the Fourth Symposium on Current Research in Muscular Dystrophy, London, 1968, pp. 255-263. Edited by $N$ the Research Committee of the Muscular Dystrophy Group. Pitman: London.

Smith, B. (1969). Skeletal muscle necrosis associated with carcinoma. Journal of Pathology, 97, 207-210.

Urich, H., and Wilkinson, M. (1970). Necrosis of muscle with carcinoma: myositis or myopathy? Journal of Neurology, Neurosurgery, and Psychiatry, 33, 398-407. 